\title{
Research on Adsorption Performance of Graphene Oxide and Its Composites for Copper Ion
}

\author{
Zongxuan Han ${ }^{*}$, Jiakun Li \\ College of Chemistry, Liaoning University, Shenyang, 110036, China \\ * Corresponding Author
}

Keywords: Adsorption performance, Copper ion, Graphene oxide

\begin{abstract}
Graphene oxide / chitosan composite material is a new type of biological composite material developed in recent years, which has unique adsorption properties. In this paper, GO/CS composite adsorbent was prepared by combining GO liquid with pretreated chitosan CS. The adsorption of copper ions of GO/CS composite was also studied. The results showed that the best conditions of the composite adsorption were $\mathrm{pH}=9$, 20mg dosage and $10 \mathrm{~min}$ adsorption time. Under such conditions, the adsorption rate is as high as $89 \%$. The paper can provide some references for the research of the chemical properties of the graphene oxide composites.
\end{abstract}

\section{Introduction}

Graphene is a monatomic planar structure formed by sp2+ hybridized carbon atoms, and is the only atomic crystal found in two-dimensional free states. In 2004, humans succeeded in obtaining the graphene. Its structure, preparation methods, properties and applications have attracted great attention both at home and abroad. Graphene super high specific surface area, excellent mechanical, thermal and electrical properties of the materials with high strength and high-performance adsorption materials, energy storage, super conductive and biomedical materials field has great application potential. Graphene oxide is a graphene derivative, usually made by oxidation of natural graphite and ultrasonic dispersion. Compared with graphene, graphene oxide has good dispersion, hydrophilicity and compatibility. Therefore, graphene oxide can form stronger interfacial interaction with the polymer matrix, and can also be intercalated or peeled by small molecules. It can be added into polymer as Nano enhancement component, which can improve the mechanical, thermal and electrical properties of polymers effectively. It is the product of deacetylation of chitin, which has a wide range of sources, non-toxic, easy to degrade and so on. The molecular structure of chitosan containing large amounts of amino and hydroxyl groups of carboxyl group, etherification, esterification, alkylation, acylation and halogenation etc. chemical reaction of chitosan and chitosan derivatives were prepared by different, giving it more special function. The chitosan also has excellent film forming ability, antibacterial property, good biodegradability and biocompatibility, so it plays an irreplaceable role in the preparation and modification of composite materials. In recent years, with the development of research, many researchers try to react with chitosan and graphene oxide, and prepare various kinds of chitosan / graphene oxide composites by different methods. This composite material combines the excellent properties of chitosan and graphene oxide, and has excellent performance in the adsorption of copper ions. This paper will study its adsorption ability of copper ion through experiment.

\section{Materials and Methods}

Materials. Atomic absorption spectrometer (Beijing Purkinje General Instrument Co., Ltd.); vacuum drying box (DZF-205), the Great Wall Zhengzhou Branch Trade and Industry Co. Ltd; electronic balance (JA1003N), Shanghai precision Scientific Instruments Inc; ultrasonic cleaner in Kunshan city (K300DA), ultrasonic instrument company; circulating water vacuum pump 
(SHZ-Dlll), Yuhua Instrument Co. company in Jinan; high speed refrigerated centrifuge (H165, Changsha Xiangyi Centrifuge Instrument Co. Ltd.); graphite powder (Hua Xuechun), Sinopharm Chemical Reagent Co. Ltd.; copper sulfate, sodium hydroxide, potassium chloride, anhydrous ethanol, sulfuric acid, hydrogen peroxide, Potassium Permanganate, were of analytical grade were from medicine group Reagent Co. ltd.. UV-752 UV visible spectrophotometer (Shanghai Phoenix Optical Instrument Co. Ltd.); AA240 flame atomic absorption spectrometer (Chinese Varian Co. Ltd.); LGJ-12 freeze dryer (Beijing Huaxing Technology Development Co. Ltd.); ThZ-320 type constant temperature oscillator (Shanghai Jinghong Experimental Equipment Co. Ltd.).

Preparation Method of Graphene Oxide and Its Composites. The preparation process of Hummers is relatively good, and the preparation process is relatively safe. It is the most common preparation method of the Graphene Oxide at present. It adopts Potassium Permanganate and graphite powder in concentrated acid by oxidation reaction, get brown material at the edges in the plane and the carboxylic derivative is mainly graphite flakes of phenolic hydroxyl and epoxy groups. The graphene layer can by ultrasound or high shear stir stripping of graphene oxide, and stable, brownish yellow single layer graphene oxide suspension formed in water. As the conjugated network is seriously functionalized, graphene oxide flakes have an insulating property. After reduction, partial reduction can be carried out to obtain chemically modified graphene flakes. Although graphene final products or reduction of graphene oxide has many defects, leading to the conductivity of graphene is better than the original, but the process of oxidation - reduction and stripping can effectively make graphite powder insoluble in water can become processing, provide the way to make a reduction of graphene oxide. In addition, the simple process and its solution process ability, considering the industrial process of mass production, the above process has become an attractive process for the manufacture of graphene related materials and components. The modified Hummers oxidation graphite specific operation is as follows: we collect $2.00 \mathrm{~g}$ graphite powder to add it into the concentrated sulfuric acid containing $35 \mathrm{~mL}$, stirring evenly and slowly add $6 \mathrm{~g} \mathrm{KMnO}_{4}$ for two hours; we control the mixture maintained at a temperature below 293K, and then placed in a water bath to keep the $4 \mathrm{~h} 308 \mathrm{~K}$ after adding $90 \mathrm{~mL}$ slowly diluted in deionized water and stir well for one hour. We can get dark brown suspension. We gradually add $30 \% \mathrm{H}_{2} \mathrm{O}_{2}$ until the color is bright yellow to pump the filtration while it is hot. Wash it by $5 \%$ hydrochloric acid washing, removing the manganese salt, and the solution is neutral. The GO of solid state can be obtained by the ultrasonic dispersing and the freeze drying.

Adsorption Experiment Method. The chemical adsorption has selection characteristic. Some adsorbent adsorbate only has certain adsorption for some materials. The chemical reaction heat is almost in the same order of magnitude, the adsorption rate and desorption rate of it is very small, but with the increase of the temperature increasing rate of adsorption. Such adsorption generally requires a certain activation energy, the adsorbed molecules and the adsorption surface and the interaction between atoms in the compound force similar. This adsorption is essentially a chemical reaction. The adsorption experiment of $\mathrm{Cu}$ adsorption experiments was carried out in a conical flask. We added the

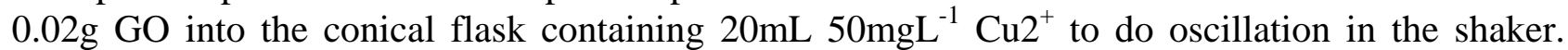
Collect each sampling $2 \mathrm{~mL}$ respectively after 0, 2, 10, 30, 60, 90, 120, 150 mins; then the sample dilution with membrane filtration. The filtrate determination by flame atomic absorption spectrometry of $\mathrm{Cu}$ liquid concentration, which has the adsorption capacity to evaluate the adsorption properties of GO on $\mathrm{Cu}$. The preparation of standard solution concentration by using copper sulfate as simulated water with copper, the concentration of $\mathrm{Cu}^{2+}$ was prepared with copper standard solution $100 \mathrm{gmL}$. Adsorption experiments were carried out to remove a certain volume of copper standard solution to $50 \mathrm{~mL}$ volumetric flask. $\mathrm{pH}$ regulation was done by diluting the $\mathrm{HCl}$ and $\mathrm{NaOH}$ solution. We add GO/CS composite material with certain quality as adsorbents for 10min oscillator shock filtering, determining the supernatant by flame atomic absorption. The measured parameters of the absorption line wavelength were $324 \mathrm{~nm}$. 


\section{Results and Analysis}

Influence of pH of Solution on Adoption Effect. There are many factors affecting the adsorption of solids in solution. They can generally be considered in terms of the relation among the three solutes, solvents and adsorbents. Some of the electrolyte has molecular state adsorption. The adsorption of small molecules is similar; some solid materials can absorb in neutral salt solution. The $\mathrm{pH}$ of the solution changes occurred as hydrolysis of salts and the solid selective adsorption changes. The adsorption is called hydrolysis adsorption; some direct and some ion solid skeleton the exchange. The two exchanges lie in the solid adsorption process which can be called ion exchange adsorption. We use the colorless phenolphthalein solution drops into acidic or neutral solution. The color will not change; the colorless phenolphthalein solution drops into alkaline solution, and the solution turns red. When the $\mathrm{pH}$ indicator is added into the colored solution, we should select the $\mathrm{pH}$ indicator which can produce obvious color difference. We respectively take 5 parts of $2.0 \mathrm{ml} \mathrm{Cu}^{2+}$ standard solution into $50 \mathrm{~mL}$ volumetric flasks with diluting $\mathrm{HCl}$ and $\mathrm{NaOH}$ solution to adjust the different values of $\mathrm{Ph}$. We added 20mg GO/CS composite materials, shocked for 30min filtration to observe influence of $\mathrm{pH}$ of solution on adoption effect. The results are shown as follows:

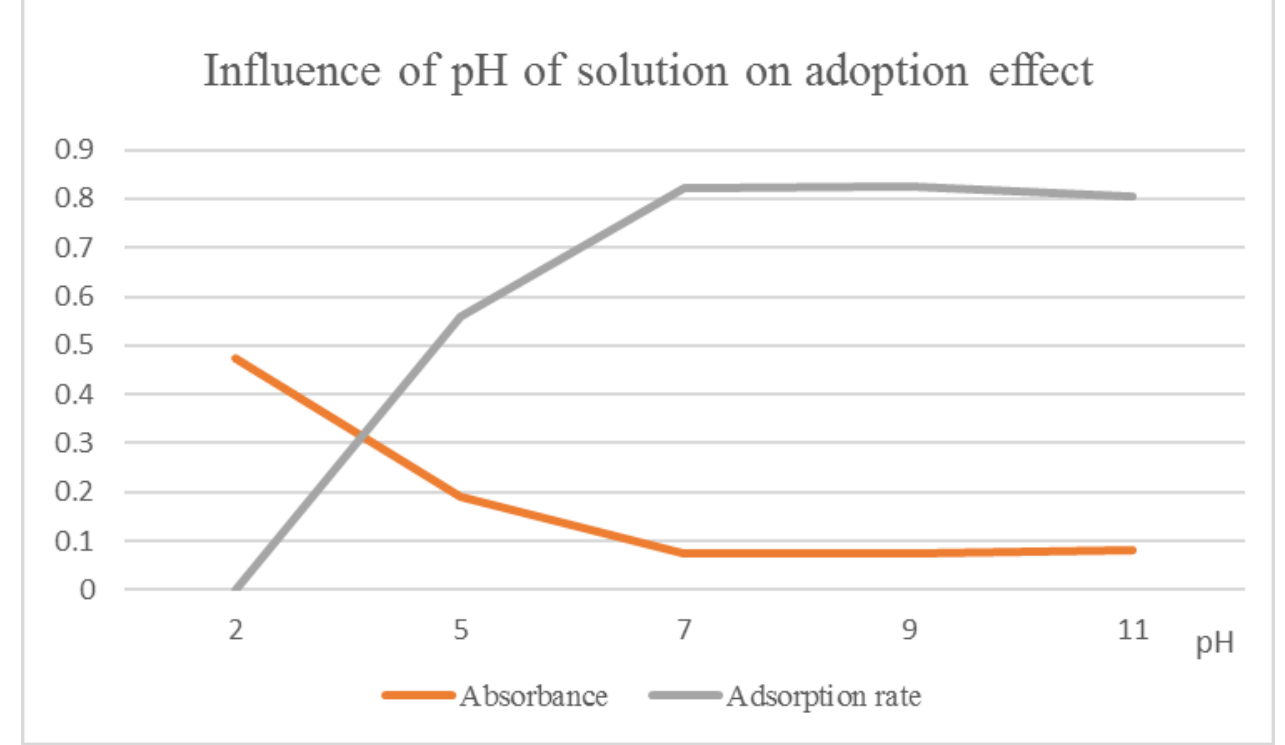

Figure 1. Figure of influence of $\mathrm{pH}$ of solution on adoption effect

Influence of Adsorption Time on Adoption Effect. On one hand, when the adsorbate and adsorbent are fully contacted, the adsorbate is absorbed by the adsorbent. On the other hand, some adsorbed adsorbate can be separated from the adsorbent surface and returned to the fluid phase as a result of thermal motion. When the adsorption rate and desorption rate is equal to the number of adsorption unit time is equal to the amount of desorption, it will no longer change in the concentration of the adsorbate and adsorbent fluid phase on the surface, this is called adsorption equilibrium. The adsorption equilibrium determines the direction and the limit of the adsorption process, and is the basic basis of the adsorption process. The adsorption rate is mainly related to particle size and pore size distribution. The amount of microspore is large, and the internal diffusion has a greater influence on the adsorption rate, and the adsorbate adsorbed on the pore wall is more. The standard solution of $\mathrm{Cu}^{2+}, \mathrm{NaOH}$ of $\mathrm{pH}=9$ were added to $20 \mathrm{mg}$ solution to adjust the GO/CS. The adsorbents oscillation was done for 5 and 10 mins and the filtering was done for 20, 30 and 40mins. The absorbance of the supernatant was measured. When the adsorption time was 10 mins, the absorbance of the solution with the lowest adsorption has the best adsorption effect. When the oscillation time was over 20 mins, the absorbance $\mathrm{Cu}^{2+}$ gradually increased. It has reached the adsorption equilibrium in the absence of oscillations, which will continue to gradually decrease. Therefore, the adsorption desorption time selected is appropriate for the $10-20 \mathrm{~min}$. The adsorption requires a certain contact time between quality and adsorption agent, which can make the maximum adsorption capacity of the adsorbent. 


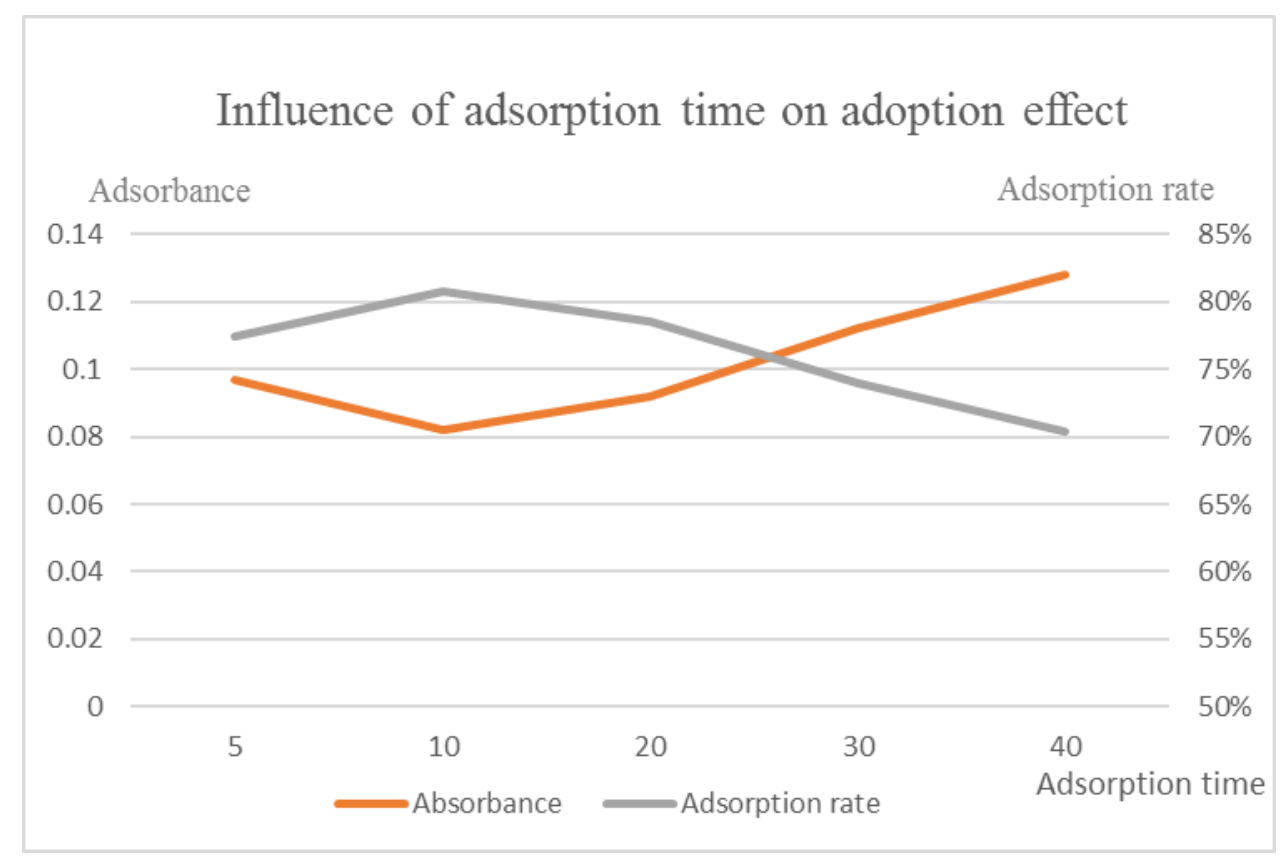

Figure 2. Figure of influence of adsorption time on adoption effect

Influence of Adsorbents Weight on Adoption Effect. We selected five standard solutions with $\mathrm{Cu}^{2+}$ in $\mathrm{NaOH}$ to adjust the $\mathrm{pH}=9$ respectively including $\mathrm{GO} / \mathrm{CS}$ composite. The filtration of the supernatant is measured after 10 mins of absorbance oscillation. When the $\mathrm{pH}$ value of adsorption time is the similar and the adsorbent dosage is 5-20mg, the absorbance decreased and the adsorption rate increased gradually. But the adsorbent quantity is $20 \mathrm{mg}$, the absorbance is minimum at this time and the adsorption rate is the maximum of GOCS. We selected the amount of composite materials for $20 \mathrm{mg}$ and the adsorbent in water with copper in the solubility of $0.4 \mathrm{mgmL}$. The smaller the solubility, the easier it is to adsorb. The size and chemical structure of adsorbate molecules also have great influence on adsorption. The adsorption rate is affected by the internal diffusion velocity, and the size of adsorbate molecules is proportional to pore size, which is most favorable for adsorption. As the adsorption phenomenon occurs on the surface of adsorbent, the specific surface area of adsorbent is one of the important factors affecting adsorption.

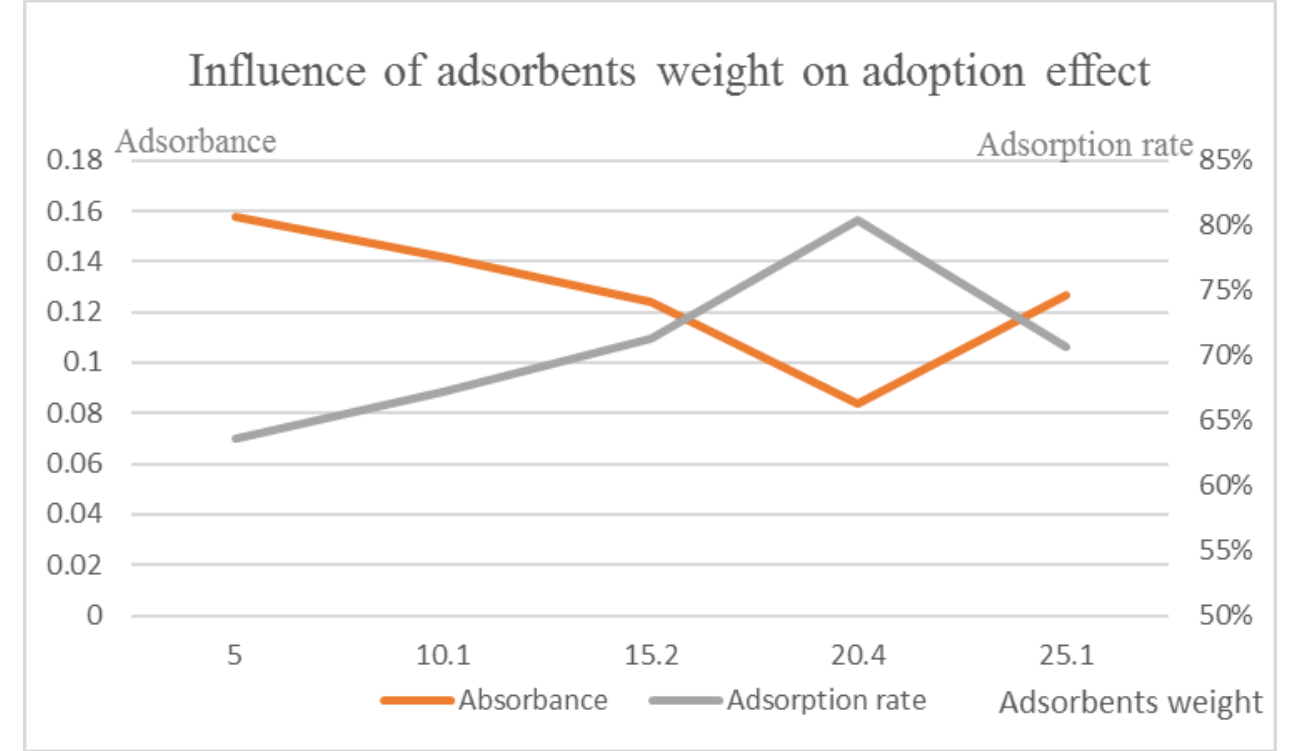

Figure 3. Figure of influence of adsorbents weight on adoption effect 


\section{Conclusions}

As a unique chemical composite, researches have made great progress in its preparations and applications in the last few years. This paper explores the Hummers method to prepare graphene oxide / chitosan composite material, and the optimum conditions of adsorption of copper ion by graphene oxide / chitosan composite are obtained through adsorption experiments to provide some references for the relatitve researchers.

\section{References}

[1] He Qiong, Qi Xiuxiu, Zhao Huanying, Fan Shuainan. Study on the preparation and application of copper ion adsorption on graphene oxide/ chitosan composite material [J]. New Chemical Materials, 2016, 44(10): 141-143.

[2] Haoran Zhao, Shenghua Lv. Review on the Preparation of Graphene Oxide and Graphene OxidePolymer Composites [J]. Polymer Materials Science and Engineering, 2016, 32(3): 184-190.

[3] Yingyue Zhang, Evan Mintzer, Kathryn E. Uhrich, Synthesis and Characterization of PEGylated Bolaamphiphiles with Enhanced Retention in Liposomes [J]. Journal of Colloid and Interface Science, 2016(482): 19-26.

[4] Li Chengyang, Zhuang Zechao, Jin Xiaoying, Chen Zuliang. Coadsorption behavior of methylene blue and $\mathrm{Cu}$ ( II ) from aqueous solution on graphene oxide [J]. Acta Scientiae Circumstantiae, 2015, 35(10): 3163-3169. 